Agrotrópica 32(3): 233 - 238. 2020.

Centro de Pesquisas do Cacau, Ilhéus, Bahia, Brasil

\title{
CARACTERÍSTICAS BIOMÉTRICAS E PRODUTIVIDADE DE PLANTAS DE MANDIOCA CULTIVAR BRS PURUS SUBMETIDAS A DIFERENTES ESPAÇAMENTOS DE PLANTIOS EM MANAUS-AM
}

\author{
Ronaldo Ribeiro de Morais*, Inocêncio Júnior de Oliveira e José Roberto Antoniol Fontes \\ Embrapa Amazônia Ocidental, Rodovia AM 010, km 29, Caixa Postal 319, 69010-970, Manaus, AM. \\ ronaldo.morais@embrapa.br; inocencio.oliveira@embrapa.br; jose.roberto@embrapa.br \\ *Autor para correspondência: ronaldo.morais@embrapa.br
}

\begin{abstract}
$\mathrm{O}$ arranjo espacial de plantio, e consequentemente, a densidade populacional de plantas tem influência direta no crescimento, desenvolvimento e produtividade de mandioca. $\mathrm{O}$ trabalha analisa os efeitos de diferentes arranjos espaciais de plantios sobre as características biométricas e de produtividade da cultivar de mandioca BRS Purus cultivada em área de terra firme em Manaus-AM. Foram avaliados seis tipos de arranjos espaciais, sendo três plantios em linhas simples, com espaçamento de 1,0 m entre as linhas e 0,6, 0,8 e 1,0 m entre as plantas, e três em linhas duplas, com espaçamentos de 2,0 m entre as fileiras duplas, $0,5,0,6$ e $0,75 \mathrm{~m}$ entre as fileiras simples e 0,5, 0,6 e $0,75 \mathrm{~m}$ entre as plantas nas fileiras simples. As variáveis biométricas avaliadas foram o diâmetro, comprimento e número de hastes e a altura das plantas, e as variáveis de produtividade avaliadas foram a massa fresca da raiz, das folhas, da parte aérea total, o número de raízes e o índice de colheita. Todos os caracteres de produção de biomassa da parte aérea, e suas repartições, apresentaram correlação significativa positiva com o caractere de produção de biomassa radicular. Os parâmetros biométricos de crescimento da cultivar BRS Purus com exceção da altura das plantas, não sofreram influência em função dos espaçamentos de plantio, e as maiores produtividade de massa fresca de raízes por área foram obtidas nas duas maiores densidades de plantio quando em fileiras simples.
\end{abstract}

Palavras-chave: Manihot esculenta, densidade de plantio, produção de raízes, massa fresca.

\section{Biometric characteristics and productivity of cassava plants cultivate BRS Purus submitted to different planting spacing in Manaus-AM. The spatial arrangement of} planting, and consequently the population density of plants has a direct influence on the growth, development and productivity of cassava. The work analyzes the effects of different spatial arrangements of planting on the biometric characteristics and productivity of the cassava cultivar BRS Purus cultivated in an area of terra firme in ManausAM. Six types of spatial arrangements were evaluated, being three plantations in simple lines, with spacing of 1.0 $\mathrm{m}$ between the lines and $0,6,0,8$ and $1,0 \mathrm{~m}$ between the plants, and three in double rows, with spacing of 2,0 $\mathrm{m}$ between double rows, $0,5,0,6$ and $0,75 \mathrm{~m}$ between single rows and $0,5,0,6$ and $0,75 \mathrm{~m}$ between plants in single rows. The biometric variables evaluated were the diameter, length and number of stems and the height of the plants, and the productivity variables evaluated were the fresh mass of the root, the leaves, the total aerial part, the number of roots and the harvest index. All the biomass production characters of the aerial part, and their distribution, showed a significant positive correlation with the root biomass production character. The biometric parameters of growth of the BRS Purus cultivar, with the exception of the height of the plants, were not influenced by the planting spacing, and the highest productivity of fresh root mass per area was obtained in the two highest planting densities when in single rows.

Key words: Manihot esculenta, planting density, root production, fresh mass. 


\section{Introdução}

No Amazonas, o cultivo de mandioca é destinado principalmente à produção de farinha, com estimativa de consumo per capita de $43,4 \mathrm{~kg}^{\text {ano-1 }}{ }^{-1}$, além do consumo in natura. Neste caso, exerce um importante papel no regime nutricional, devido ao seu alto valor energético, sobretudo entre as classes de menor poder aquisitivo (Dias, 2015).

Apesar da relevância da cultura da mandioca para o estado do Amazonas, a produtividade média alcançada em 2017 foi de apenas 9,65 tha ${ }^{-1}$. A baixa produtividade dos cultivos de mandioca no estado do Amazonas se dá pelo manejo inadequado da cultura, incluindo-se a ineficiente nutrição da planta, falta de controle inadequado de plantas infestantes, etc, e principalmente pelo uso de cultivares tradicionais de baixa produtividade e de arranjo espacial com população de plantas abaixo do recomendado para a cultura no Estado, geralmente de $1 \mathrm{~m} \mathrm{x} 1 \mathrm{~m}$ e 10.000 plantas por hectare. Em contrapartida, com o plantio de mandioca, no espaçamento de $1 \mathrm{~m} \times 1 \mathrm{~m}$ de cultivares recomendadas, como BRS Purus, que possui raiz de polpa creme e teor de amido de $26,0 \%$, pode-se atingir a produtividade de 25,0 $\mathrm{tha}^{-1}$ aos 12 meses de ciclo (Dias et al., 2004).

Os estudos sobre arranjos espaciais com adensamentos de plantas são importantes para estimar os índices de ganho de produtividade até certo nível populacional. A mandioca comumente é plantada em cultivo solteiro em linhas simples, pois o adensamento proporciona maiores rendimentos à cultura por área plantada (Mattos et al., 2005, Gabriel Filho, Strohhaecker e Fey, 2003). Contudo, quando há a necessidade de consorciar a mandioca com outras culturas, tem sido utilizado o plantio em fileiras duplas, com espécies como milho, feijão caupi e soja (Devide et al., 2009; Mattos et al., 2005; Tsay, Fukay e Wilson,
1987). No sistema de plantio de fileiras duplas existe a vantagem dos tratos culturais e rotação de culturas na mesma área, mantendo-se a produtividade (Mattos, Souza e Caldas, 1990, Mattos et al., 2005, Gabriel Filho, Strohhaecker e Fey, 2003; Silva et al., 2013; Rojas et al., 2007).

O trabalho analisa os efeitos de diferentes arranjos espaciais de plantios sobre as características biométricas e de produtividade da cultivar de mandioca BRS Purus cultivada em ambiente de terra firme em Manaus-AM.

\section{Material e Métodos}

O trabalho foi conduzido no campo experimental da Embrapa Amazônia Ocidental, em Manaus, AM (02 $53^{\prime} 48^{\prime \prime}$ S, 59 59' $59^{\prime \prime}$ 'W, 102 m de elevação), com clima do tipo Af, em Latossolo Amarelo, distrófico, muito argiloso. As condições climáticas em relação à precipitação e temperatura média do ar foram monitoradas durante o período total do experimento (Figura 1).

A temperatura mínima não foi inferior a $24^{\circ} \mathrm{C}$ e a máxima, não ultrapassou os $28^{\circ}$. A precipitação acumulada durante o período do experimento foi de $2736 \mathrm{~mm}$, com as maiores taxas reportadas para fevereiro $(377 \mathrm{~mm})$ e as menores observadas em

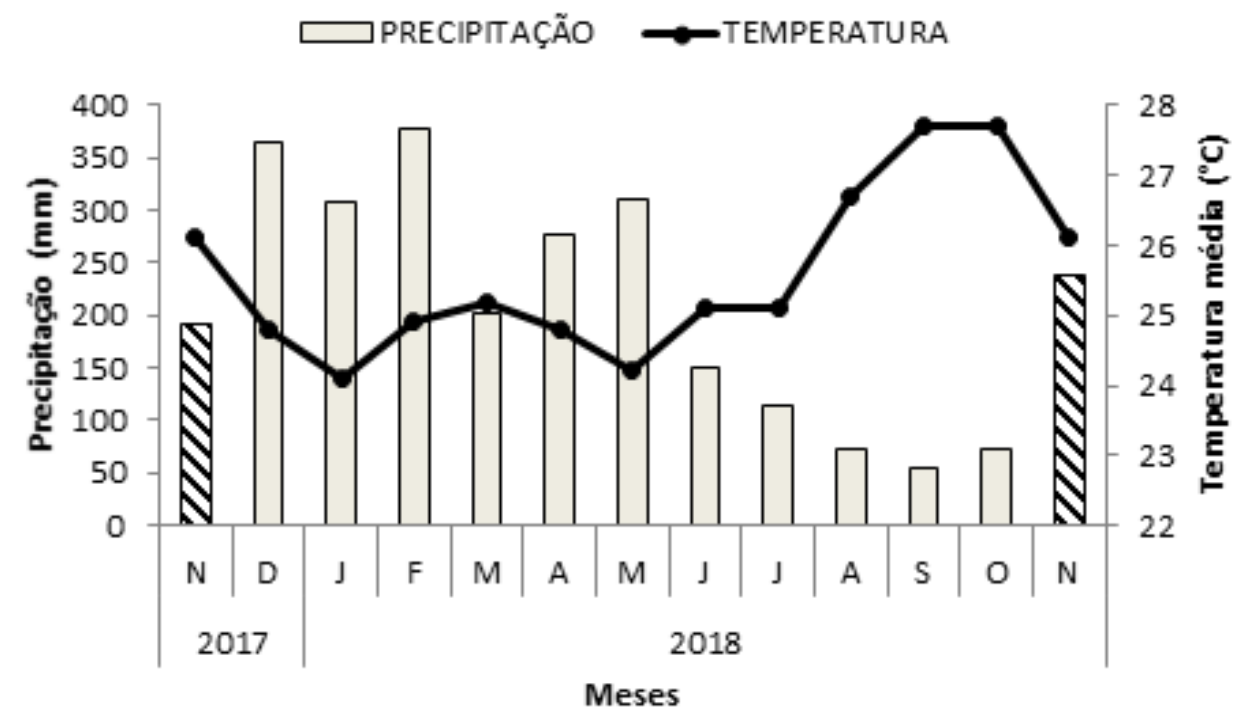

Figura 1. Dados climatológicos de precipitação mensal $(\mathrm{mm})$ e temperatura média do $\operatorname{ar}\left({ }^{\circ} \mathrm{C}\right)$ obtidos na estação agrometereológica do Campo Experimental da Embrapa Amazônia Ocidental, em Manaus, AM. Em destaque, hachurados no gráfico, os dados de precipitação obtidos no período do plantio (novembro-2017) e da colheita (novembro-2018). 
agosto (72 mm). Como observado na Figura 1, o plantio foi realizado no início do período mais chuvoso na região, com as condições adequadas em relação ao status hídrico do solo, o que propiciou um crescimento e desenvolvimento das plantas de mandioca, sem que houvesse restrição hídrica para as plantas. Já a colheita foi realizada um ano após o plantio, no início do período das chuvas na região.

O preparo de solo foi realizado com arado de discos, a uma profundidade de $40 \mathrm{~cm}$ e grade niveladora para eliminação de torrões e o plantio foi realizado em Novembro de 2017, utilizando-se manivas-sementes com $20 \mathrm{~cm}$ de comprimento da cultivar BRS Purus, que apresentar características de crescimento com ramificação de hábito dicotômico, formato da raiz cônica cilíndrica, teor de amido médio de $26 \%$ e de ciclo de 12 a 16 meses (Dias, Xavier e Barreto, 1999)

Na ocasião do plantio, os sulcos foram abertos com sulcadora-adubadora, à profundidade média de $10 \mathrm{~cm}$, seguindo os espaçamentos de cada tratamento, e a adubação de plantio foi realizada com a aplicação 80 $\mathrm{kg} \mathrm{ha}^{-1}$ de $\mathrm{P}_{2} \mathrm{O}_{5}$ na forma de superfosfato simples. Foi realizada a adubação de cobertura com $300 \mathrm{~kg} \mathrm{ha}^{-1}$ da fórmula 18-00-18 para fornecimento de $\mathrm{N} \mathrm{e} \mathrm{K}_{2} \mathrm{O}$, sendo a metade da dose aos 30 dias após o plantio e outra metade aos 60 dias após o plantio da mandioca.

O controle de plantas daninhas foi realizado por meio da aplicação em pré-emergência do herbicida isoxaflutole $\left(93,7 \mathrm{~g}\right.$ de i. a. ha $\left.{ }^{-1}\right)$, da aplicação em pósemergência do herbicida bentazon (960 g de i. a. ha $\left.{ }^{-1}\right)$ aos 25 e 90 dias após o plantio e mais uma capina aos 120 dias após o plantio.

Os tratamentos foram constituídos por seis arranjos espaciais de plantas, com interação entre espaçamento entre as fileiras e espaçamento entre as plantas. Para os tratamentos em fileiras simples, os tratamentos foram os seguintes para espaçamento entre fileiras $\mathrm{x}$ espaçamento entre plantas, respectivamente: $\mathrm{T} 1=1,0$ $\mathrm{m} \times 0,6 \mathrm{~m}\left(16667\right.$ plantas ha $\left.^{-1}\right), \mathrm{T} 2=1,0 \mathrm{~m} \times 0,8 \mathrm{~m}$ $\left(12500\right.$ plantas ha $\left.{ }^{-1}\right), \mathrm{T} 3=1,0 \mathrm{~m} \times 1,0 \mathrm{~m}(10000$ plantas $\left.\mathrm{ha}^{-1}\right)$, e para os tratamentos em fileiras duplas, os tratamentos foram os seguintes para o espaçamento entre as fileiras duplas $\mathrm{x}$ espaçamento entre fileiras simples $\mathrm{x}$ espaçamento entre plantas na fileira simples, respectivamente: $\mathrm{T} 4=2,0 \mathrm{~m} \times 0,5 \mathrm{~m} \times 0,5 \mathrm{~m}(16000$ plantas ha $\left.\mathrm{a}^{-1}\right), \mathrm{T} 5=2,0 \mathrm{~m} \times 0,6 \mathrm{~m} \times 0,6 \mathrm{~m}$ (12820 plantas $\left.\mathrm{ha}^{-1}\right), \mathrm{T} 6=2,0 \mathrm{~m} \times 0,75 \mathrm{~m} \times 0,75 \mathrm{~m}\left(9709\right.$ plantas ha $\left.^{-1}\right)$.
Os tratamentos foram dispostos em delineamento experimental de blocos ao acaso com três repetições. As parcelas experimentais foram compostas por quatro linhas para os tratamentos em fileiras simples e por três fileiras duplas para os tratamentos em fileiras duplas com seis metros de comprimento, perfazendo um total de plantas úteis distintas para os tratamentos $(\mathrm{T} 1$ e $\mathrm{T} 4=16$ plantas, $\mathrm{T} 2$ e $\mathrm{T} 5=12$ plantas e $\mathrm{T} 3$ e $\mathrm{T} 6=$ 10 plantas).

Para melhor detalhamento e comparação das variáveis biométricas e de produção de biomassa, em cada parcela da área útil, foram selecionadas três plantas aleatoriamente para o acompanhamento dos parâmetros: diâmetro da haste (DH) (a $10 \mathrm{~cm}$ do nível do solo); comprimento da haste principal $(\mathrm{CH})$; altura da planta (AP); número de haste $(\mathrm{NH})$; massa fresca radicular (MFR); massa fresca da parte aérea (MFPA) (hastes + folhas); massa fresca de hastes (MFH); massa fresca de folhas (MFF); número de raízes por planta (NR).

Para melhor avaliação da produtividade por área útil, foram coletadas todas as plantas das parcelas e avaliadas a massa fresca da parte aérea (MFPA), número de raízes por planta (NR), massa fresca radicular (MFR) e o índice de colheita (IC) por meio da equação (MFR/(MFR+MFPA)*100).

Os dados foram submetidos à análise de variância, e teste Tukey para a comparação de médias a $5 \%$ de probabilidade. Realizou também a análise de correlação de Pearson.

\section{Resultados e Discussão}

Para a análise de correlação de Pearson utilizaramse todos os dados independente dos tratamentos $(n=$ 54) (Tabela 1). Os dados mostram correlação significativa negativa entre o diâmetro da haste e o número de hastes $(r=-0,47)$, e que o diâmetro de haste não teve correlação significativa com os outros caracteres biométricos e de produtividade. O comprimento das hastes teve correlação significativa com o número de hastes produzidas por planta $(r=0,34)$, e também, com o número de raízes produzidas $(r=0,34)$. $\mathrm{O}$ número de raízes produzidas por plantas $(\mathrm{NR})$, não teve correlação significativa apenas com os caracteres de diâmetro da haste (DH) e altura das plantas (AP).

Contudo, o caractere biométrico que mais se correlacionou como os caracteres de produção de 
biomassa, foi o número de hastes produzidas por planta, o qual apresentou correlação significativa com MFR, MFPA, MFH, MFF e NR (Tabela 1).

Para a cultivar BRS Purus, se observa que todos os caracteres, com exceção do diâmetro da haste, comprimento da haste e altura das plantas, apresentaram correlação significativa positiva com a produção de massa fresca de raiz, mostrando a importância do crescimento e desenvolvimento da parte aérea da planta da cultivar BRS Purus sobre a produção de raízes (Tabela 1).

Em estudos de Gabriel Filho, Strohhaecker e Fey (2003), os plantios realizados em fileiras simples ou duplas, não interferiram nas variáveis biométricas estudadas. A distância entre plantas na linha não afetou a altura e o diâmetro do caule das plantas de mandioca (Rojas et al., 2007). Este resultado, em experimentos agrícolas, é de suma importância, principalmente em programas de melhoramento de plantas, assim como, em estudos dos diferentes arranjos espaciais de plantios visando possibilidades de plantios mistos com outras culturas.

Analisando-se os resultados da comparação de médias entre os tratamentos para os caracteres biométricos e de produção de biomassa referentes a plantas selecionadas aleatoriamente nas parcelas $(n=9)$ (Tabela 2), observa-se que somente a característica biométrica de altura da planta apresentou diferenças entre os tratamentos, onde as plantas de mandioca do T5 apresentaram as maiores alturas em relação aos $\mathrm{T} 1, \mathrm{~T} 2$ e T3. Isto pode ser resultado da pouca

Tabela 1. Matriz de coeficientes de correlação linear de Pearson entre caracteres de crescimento e produtividade (Diâmetro da haste principal - DH; Comprimento da haste principal - CH; Altura da planta - AP; Número de hastes - NH; Massa fresca de raiz - MFR; Massa fresca da parte aérea - MFPA; Massa fresca de hastes - MFH; Massa fresca de folhas - MFF e Número de raízes - NR) da mandioca

\begin{tabular}{|c|c|c|c|c|c|c|c|c|c|}
\hline & DH & CH & AP & NH & MFR & MFPA & MFH & MFF & NR \\
\hline DH & - & $-0.20^{\text {ns }}$ & $0,24^{\mathrm{ns}}$ & $-0,47 * *$ & $-0,00^{\text {ns }}$ & $0,01^{\mathrm{ns}}$ & $0,04^{\mathrm{ns}}$ & $-0,13^{\mathrm{ns}}$ & $-0,05^{\text {ns }}$ \\
\hline CH & & - & $0,25^{\mathrm{ns}}$ & $0,34 *$ & $0,25^{\mathrm{ns}}$ & $0,24^{\mathrm{ns}}$ & $0,25^{\mathrm{ns}}$ & $0,08^{\mathrm{ns}}$ & $0,31^{*}$ \\
\hline $\mathbf{A P}$ & & & - & $-0,06^{\mathrm{ns}}$ & $0,06^{\mathrm{ns}}$ & $0,21^{\mathrm{ns}}$ & $0,21^{\mathrm{ns}}$ & $0,12^{\mathrm{ns}}$ & $0,12^{\mathrm{ns}}$ \\
\hline NH & & & & - & $0,42 * *$ & $0,48 * *$ & $0,47 * *$ & $0,30 *$ & $0,57 * *$ \\
\hline MFR & & & & & - & $0,79 * *$ & $0,78 * *$ & $0,50 * *$ & $0,67 * *$ \\
\hline MFPA & & & & & & - & $0,98 * *$ & $0,63 * *$ & $0,67 * *$ \\
\hline MFH & & & & & & & - & $0,51 * *$ & $0,66 * *$ \\
\hline MFF & & & & & & & & - & $0,42 * *$ \\
\hline NR & & & & & & & & & - \\
\hline
\end{tabular}

Tabela 2. Comparação de médias do diâmetro da haste $(\mathrm{DH})$, comprimento da haste $(\mathrm{CH})$, altura da planta (AP), número de hastes (NH), massa fresca da raiz (MFR), massa fresca da parte aérea (MFPA), massa fresca das hastes (MFH), massa fresca das folhas (MFF) e número de raízes (NR), entre os tratamentos (TRAT) dos diferentes arranjos espaciais do plantio de mandioca (T1 $(1,0 \mathrm{~m} \times 0,6 \mathrm{~m}) ; \mathbf{T} 2(1,0 \mathrm{~m} \times 0,8 \mathrm{~m}) ; \mathbf{T 3}(1,0 \mathrm{~m} \times 1,0 \mathrm{~m}) ; \mathbf{T} 4(2,0 \mathrm{~m} \times 0,5 \mathrm{~m} \times 0,5 \mathrm{~m}) ; \mathbf{T 5}(2,0 \mathrm{~m} \times 0,6 \mathrm{~m} \times 0,6 \mathrm{~m})$ e T6 (2,0 m x 0,75 $\mathrm{m} \times 0,75 \mathrm{~m})), n=9$

\begin{tabular}{|c|c|c|c|c|c|c|c|c|c|}
\hline TRAT & $\begin{array}{l}\text { DH } \\
(\mathrm{cm})\end{array}$ & $\begin{array}{l}\mathrm{CH} \\
(\mathrm{cm})\end{array}$ & $\begin{array}{l}\text { AP } \\
(\mathbf{m})\end{array}$ & $\begin{array}{l}\text { NH } \\
\text { (un) }\end{array}$ & $\begin{array}{c}\text { MFR } \\
\left(\mathrm{kg} \mathrm{pl.} .^{-1}\right)\end{array}$ & $\begin{array}{c}\text { MFPA } \\
\left(\mathrm{kg} \mathrm{pl.}^{-1}\right)\end{array}$ & $\begin{array}{c}\text { MFH } \\
\left(\mathrm{kg} \mathrm{pl}^{-1}\right)\end{array}$ & $\begin{array}{c}\text { MFF } \\
\left(\mathrm{kg} \mathrm{pl.}^{-1}\right)\end{array}$ & $\begin{array}{l}\text { NR } \\
\text { (un) }\end{array}$ \\
\hline $\mathrm{T} 1$ & $2,46 \mathrm{a}$ & $52,33 \mathrm{a}$ & $2,08 \mathrm{bc}$ & $2,22 \mathrm{a}$ & $5,13 \mathrm{a}$ & $2,98 \mathrm{a}$ & $2,55 \mathrm{a}$ & $0,42 \mathrm{a}$ & $7,56 \mathrm{a}$ \\
\hline $\mathrm{T} 2$ & $2,74 \mathrm{a}$ & $57,67 \mathrm{a}$ & $2,07 \mathrm{bc}$ & $1,89 \mathrm{a}$ & $4,80 \mathrm{a}$ & $3,11 \mathrm{a}$ & $2,82 \mathrm{a}$ & $0,28 \mathrm{a}$ & $7,22 \mathrm{a}$ \\
\hline $\mathrm{T} 3$ & $2,66 \mathrm{a}$ & $34,67 \mathrm{a}$ & $1,98 \mathrm{c}$ & $1,78 \mathrm{a}$ & $4,97 \mathrm{a}$ & $3,44 \mathrm{a}$ & $3,15 \mathrm{a}$ & $0,28 \mathrm{a}$ & $6,44 \mathrm{a}$ \\
\hline $\mathrm{T} 4$ & $2,74 \mathrm{a}$ & $59,78 \mathrm{a}$ & $2,31 \mathrm{ab}$ & $1,33 \mathrm{a}$ & $4,25 \mathrm{a}$ & $3,15 \mathrm{a}$ & $2,75 \mathrm{a}$ & $0,40 \mathrm{a}$ & $5,89 \mathrm{a}$ \\
\hline $\mathrm{T} 5$ & $2,78 \mathrm{a}$ & $51,22 \mathrm{a}$ & $2,39 \mathrm{a}$ & $1,33 \mathrm{a}$ & $4,04 \mathrm{a}$ & $3,14 \mathrm{a}$ & $2,79 \mathrm{a}$ & $0,34 \mathrm{a}$ & $6,33 \mathrm{a}$ \\
\hline T6 & $2,73 \mathrm{a}$ & $54,11 \mathrm{a}$ & $2,27 \mathrm{ab}$ & $1,67 \mathrm{a}$ & $5,11 \mathrm{a}$ & $3,32 \mathrm{a}$ & $3,03 \mathrm{a}$ & $0,29 \mathrm{a}$ & $6,67 \mathrm{a}$ \\
\hline Média & 2,67 & 51,63 & 2,18 & 1,70 & 4,72 & 3,19 & 2,85 & 0,33 & 6,68 \\
\hline CV \% & 9,94 & 44,20 & 4,50 & 27,62 & 20,30 & 19,19 & 18,58 & 46,91 & 19,01 \\
\hline
\end{tabular}

Médias seguidas pela mesma letra na coluna não diferem pelo teste Tukey a 5\% de probabibilidade. 
variabilidade do comportamento biológico da cultivar BRS Purus em função dos distintos espaçamentos, ou devido o número de plantas insuficiente por tratamento (9 plantas) que pode ter tido efeito para que as diferenças não tenham sido significativas.

Essa possibilidade de amostragem insuficiente para a ausência de diferenças entre os parâmetros biométricos e de produtividade entre os tratamentos é corroborada pelos resultados de produtividade em relação à toda área útil, pois com exceção do número de raízes por planta, as demais variáveis de produtividade apresentaram diferenças entre os tratamentos (Figura 2).

O tratamento 6 , em fila dupla com a menor densidade de plantio ( 9.709 plantas/ha) apresentou a menor produção de biomassa de raízes em comparação aos demais tratamentos. Em estudos realizados por Bueno (1986) em plantios de dez cultivares de mandioca, plantadas em fileiras simples e duplas, verificou-se a maior eficiência na produtividade das cultivares plantadas nas fileiras simples, fato este decorrente do maior número de raízes por planta na fileira simples. Streck et al. (2014) estudando quatros
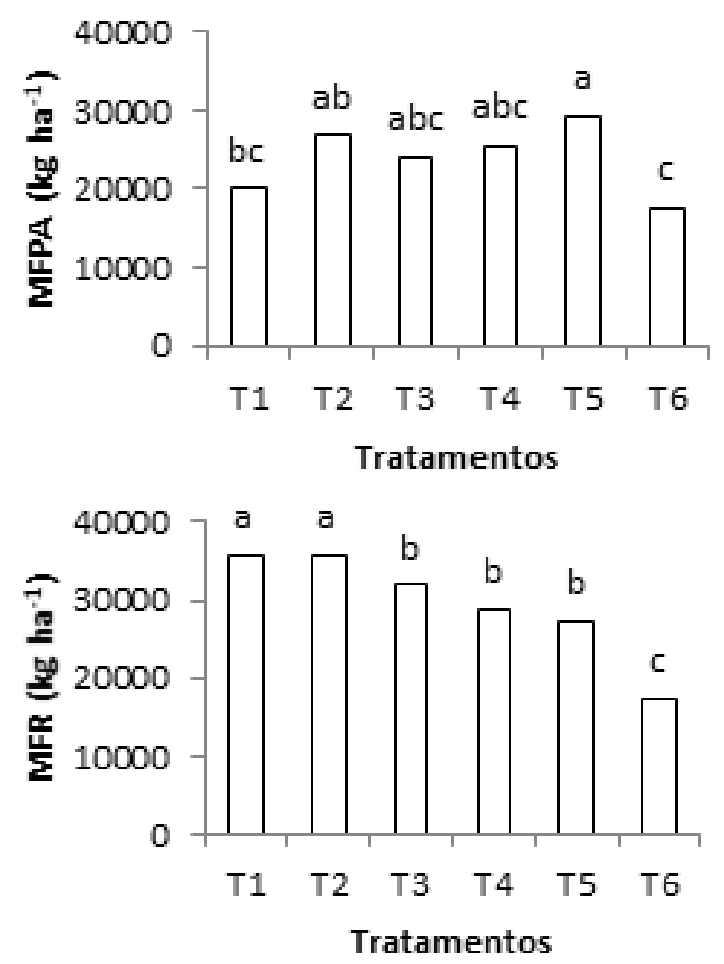

diferentes densidades de plantio para a cultivar Fepagro, verificou que a produtividade de raiz por área é maior em plantios de densidades maiores, enquanto que a produtividade por planta é maior em plantios de menor densidade, pois o investimento da planta na parte aérea seria maior nesta densidade de plantio. Isto também verificado por Mattos, Souza e Caldas (1984), onde foi observado que em menores densidades de plantio, há uma maior produtividade de planta, pois o número de raízes por planta cresce quando o espaçamento entre fileiras e dentro delas é aumentado.

A produção de raízes por área é importante para fins industriais, e que a produção de raízes por planta quando a produção é voltada para o consumo de mesa (Aguiar et al., 2011; Streck et al., 2014). Além disso, segundo Oliveira et al. (2019) o resultado da matéria seca seja mais relevante devido estar intrinsicamente associado aos processos fisiológicos, os resultados da matéria seca são importantes para se estimar a produção, com as médias regionais e nacionais, visto que a mandioca é comercializada em relação ao seu peso.
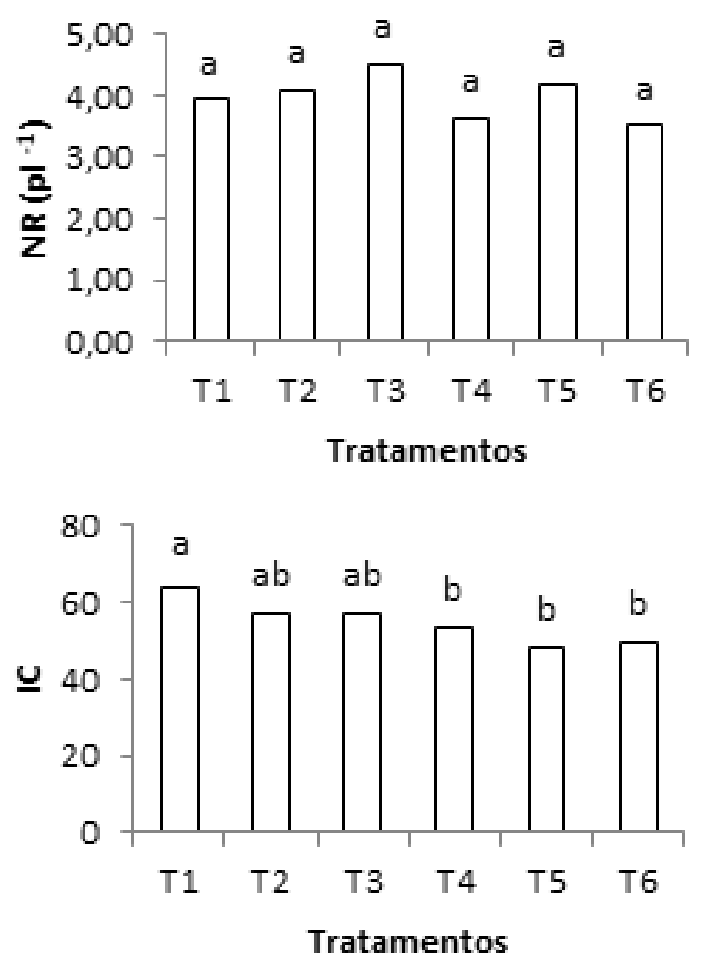

Figura 2. Comparação de médias dos parâmetros de produtividade, massa fresca da parte aérea (MFPA), número de raízes (NR), massa fresca das raízes (MFR) e índice de colheita (IC) da área útil para os diferentes arranjos espaciais de plantio de mandioca. (T1 (1,0 x 0,6); T2 (1,0 x 0,8); T3 (1,0 x 1,0); T4 (2,0 x 0,5 x 0,5); T5 (2,0 x 0,6 x $0,6)$ e T6 $(2,0 \times 0,75 \times 0,75))$. Médias seguidas pela mesma letra não diferem pelo teste Tukey a 5\% de probabibilidade. 
Em relação à densidade de plantios, observa-se que os plantios com densidades quase similares de plantas por área $(\mathrm{T} 2=12.500$ planta/ha e T5 $=12.820$ planta/ ha), a produção de massa fresca de raízes por área foi superior no plantio de fileira simples $(35.790 \mathrm{~kg} / \mathrm{ha})$ em comparação ao de fileiras duplas $(27.169 \mathrm{~kg} / \mathrm{ha})$, com uma redução de aproximadamente $24 \%$ na produtividade por área. Este fato também se repete para os tratamentos T3 (10.000 plantas/ha) e T6 (9.709 plantas/ha), onde a produtividade de massa fresca de raízes por área foi superior para o plantio em fileira simples $(31.876 \mathrm{~kg} / \mathrm{ha})$ em comparação ao plantado em fileiras duplas $(17.460 \mathrm{~kg} / \mathrm{ha})$.

$O$ índice de colheita (IC) do tratamento 1 (espaçamento $1,0 \times 1,0$ ) foi superior a todos os tratamentos em fileiras duplas, sem diferença para os demais tratamentos em fileiras simples, mostrando a maior produtividade por área nas maiores densidades de plantios para a cultivar BRS Purus (Figura 2).

Em estudos realizados por Gabriel Filho, Strohhaecker e Fey (2003) constatou-se que a produtividade de mandioca por área é maior em menores densidades de plantio, fato este explicado pela maior área de abrangência do sistema radicular, devido ao maior espaço disponível para seu crescimento. Para Guerra et al. (2003) e Rojas et al. (2007), esta produtividade em função da densidade de plantio depende muito da cultivar plantada. Em estudos de Silva et al. (2013) a densidade ideal encontrada para cultivar Vermelhinha foi de 13.594 plantas $_{\text {ha }}{ }^{-1}$, e com o aumento da densidade do plantio, constatou-se a redução no comprimento das raízes e no índice de colheita.

\section{Conclusões}

Os parâmetros biométricos de crescimento da cultivar BRS Purus, com exceção da altura das plantas não sofreram influência em função dos arranjos espaciais dos plantios.

A cultivar BRS Purus apresentou as maiores produtividades de massa fresca de raízes por área nas maiores densidades nos plantios em fileiras simples.

\section{Literatura Citada}

AGUIAR, E. B. et al. 2011. Efeito da densidade populacional e época de colheita na produção de raízes de mandioca de mesa. Bragantia 70(3):561-569.
BUENO, A. 1986. Comportamento de genótipos contrastantes de mandioca em diferentes sistemas de plantio. Pesquisa Agropecuária Brasileira 21(6):631-640.

DEVIDE, A. C. P. et al. 2009. Produtividade de raízes de mandioca consorciada com milho e caupi em sistema orgânico. Bragantia 68(1):145-153.

DIAS, M. C.; XAVIER, J. J. B. N.; BARRETO, J. F. 1999. Cultivar BRS Purus: nova alternativa de mandioca para terra firme no Amazonas. Embrapa Amazônia Ocidental, folheto.

DIAS, M. C. et al. 2004. Recomendações técnicas do cultivo de mandioca para o Amazonas. Manaus: Embrapa Amazônia Ocidental, Circular Técnica $n^{\circ} 23.24 \mathrm{p}$.

DIAS, M. C. 2015. A contribuição da pesquisaexperimentação e o conhecimento tradicional para o cultivo da mandioca no Amazonas. Terceira Margem: Amazônia 1(5):143-154.

GABRIEL FILHO, A.; STROHHAECKER, L.; FEY, E. 2003. Profundidade e espaçamento da mandioca no plantio direto na palha. Ciência Rural 33(3):461-467.

GUERRA, A. F. et al. 2003. Produtividade e qualidade de raízes de mandioca em resposta ao regime hídrico e a densidade de plantio. Planaltina, DF, Embrapa. 12p.

MATTOS, P. L. P. de; SOUZA, L. da S.; CALDAS, R. C. 1984. Sistemas de plantio de mandioca em fileira dupla no Brasil. In: Práticas Culturais da Mandioca. Salvador, BA, 1980. Anais. Brasília, DF, EMBRAPA-DDT. pp.87-94.

MATTOS, P. L. P. de; SOUZA, A. da S.; CALDAS, R. C. 1990. Consorciação de mandioca plantada em fileiras duplas comfeijão. Revista Brasileira de Mandioca 9(2):83-90.

MATTOS, P. L. P. et al. 2005. Consorciação da mandioca plantada em fileiras duplas e simples com culturas de ciclo curto. I. mandioca x caupi x milho. Revista Brasileira de Mandioca 18(1):25-30.

OLIVEIRA, E. C. et al. 2019. Produção de biomassa de mandioca com dosséis contrastantes em diferentes populações e épocas de colheita. Agrotrópica (Brasil) 31(1):53-60.

ROJAS, R. et al. 2007. Efecto de ladensidad de plantación sobre El desarrollo y rendimiento del cultivo de layuca Manihot esculenta Crantz, bajo las condiciones agroecológicas de La Altiplanicie de Maracaibo. Revista de la Facultad de Agronomia 24(1):94-112.

SILVA, T. S. et al. 2013. Planting density and yield of cassava roots. Revista Ciência Agronômica 44(2):317-324.

STRECK, N. A. et al. 2014. Efeito do espaçamento de plantio no crescimento, desenvolvimento e produtividade da mandioca em ambiente subtropical. Bragantia 73(4):407-415.

TSAY, J. S.; FUKAY, S.; WILSON, G. L. 1987. The response of Cassava (Manihot esculenta) to spatial arrangement and to soybean intercrops. Fields Crops Research 16(1):19-31. 